\title{
Efficacy and Safety of Vildagliptin as an Add-On Therapy in Inadequately Controlled Type 2 Diabetes Patients Treated With Basal Insulin
}

\author{
Daisuke Saito ${ }^{a}$, Akio Kanazawa, ${ }^{a}$, e , Nayumi Shigihara ${ }^{a}$, Fumihiko Sato ${ }^{a}$, Toyoyoshi Uchida ${ }^{a}$, \\ Junko Sato ${ }^{\text {a }}$, Hiromasa Goto ${ }^{\text {a }}$, Takeshi Miyatsuka ${ }^{\text {a }}$, Fuki Ikeda ${ }^{a}$, Takeshi Ogihara ${ }^{a}$, \\ Chie Ohmura ${ }^{a}$, Hirotaka Watada ${ }^{a}, b, c, d$
}

\begin{abstract}
Background: The aim of this study was to investigate the efficacy and safety of vildagliptin as an add-on therapy for patients with type 2 diabetes mellitus inadequately controlled with basal insulin.

Methods: Twenty-four patients treated with basal insulin and oral anti-diabetes drugs were randomly allocated into two groups: the control group (did not receive any add-on drugs) and vildagliptin group (received vildagliptin $100 \mathrm{mg}$ /day for 6 months). The primary outcome was changes in hemoglobin A1c (HbA1c) from baseline to end of study.
\end{abstract}

Results: Treatment with vildagliptin significantly reduced $\mathrm{HbA1c}$ from $8.1 \pm 0.7 \%$ at baseline to $7.1 \pm 0.7 \%(\mathrm{P}<0.01)$, while there was no significant change of $\mathrm{HbA} 1 \mathrm{c}$ in the control group. Vildagliptin group showed significant reduction of HbAlc compared with control group $(-1.0 \pm 0.3 \%$ vs. $0.2 \pm 0.8 \%, \mathrm{P}<0.01)$. In addition, vildagliptin group showed a significant increase in 1,5-anhydroglucitol compared with the control group $(4.5 \pm 3.4$ vs. $0.5 \pm 4.1 \mu \mathrm{g} / \mathrm{mL}, \mathrm{P}<0.05)$. Mild hypoglycemia was reported in one patient of the vildagliptin group and two patients of the control group.

Conclusion: Vildagliptin improved glycemic control without increasing hypoglycemia in Japanese type 2 diabetes inadequately controlled with basal insulin treatment and other oral anti-diabetes drugs. This study was registered with UMIN (University Hospital Medical Information Network ID\#000010849).

Manuscript accepted for publication December 21, 2016

aDepartment of Metabolism \& Endocrinology, Juntendo University Graduate School of Medicine, Tokyo, Japan

${ }^{b}$ Center for Therapeutic Innovations in Diabetes, Juntendo University Graduate School of Medicine, Tokyo, Japan

${ }^{\mathrm{c}}$ Center for Identification of Diabetic Therapeutic Targets, Juntendo University Graduate School of Medicine, Tokyo, Japan

${ }^{\mathrm{d} S}$ Sportology Center, Juntendo University Graduate School of Medicine, Tokyo, Japan

${ }^{e}$ Corresponding Author: Akio Kanazawa, Department of Metabolism \& Endocrinology, Juntendo University Graduate School of Medicine, 2-1-1 Hongo, Bunkyou-ku, Tokyo 113-8421, Japan. Email: akana@juntendo.ac.jp

doi: https://doi.org/10.14740/jocmr2874w
Keywords: Basal insulin; DPP-4 inhibitor; 1,5-anhydroglucitol

\section{Introduction}

The main goals of treatment of diabetes mellitus are to prevent diabetic complications and maintain good quality of life. For this purpose, intensive treatment using various oral anti-diabetes drugs (OADs) is often required in daily practice. However, due to the progressive nature of type 2 diabetes mellitus (T2DM), many T2DM patients eventually require insulin therapy to achieve better glycemic control [1]. However, patients are often reluctant to be treated with insulin for several reasons, such as increased risk of hypoglycemia, fear or inconvenience to "injection", and concerns of complex insulin regimens. For similar reasons, physicians are also reluctant to introduce insulin therapy. It is no exaggeration to say that these factors have led to delayed insulin initiation at least in Japan [2]. In contrast, treatment regimens that include the addition of long-acting basal insulin injection to ongoing treatment with OADs have been widely adopted for patients with T2DM [3]. The simple and once-daily injection is expected to reduce the psychological burden of insulin treatment and is clinically effective (more than $1.0 \%$ reduction from the baseline) [4]. However, among the patients treated with basal insulin and OADs, $73.1 \%$ patients did not achieve target hemoglobin A1c (HbAlc) level $(<7.0 \%)$ [3]. This result clearly suggests the need for additional treatment.

Dipeptidyl peptidase-4 (DPP-4) inhibitors increase the serum concentrations of glucagon-like peptide-1, which promotes glucose-response insulin secretion and inhibits glucagon secretion from $\alpha$ cells [5]. Accordingly, these drugs can decrease both fasting and postprandial glucose levels [6] with low risk of hypoglycemia and without body weight gain. Additionally, the glucose-lowering effect is known to be greater in Asians than Caucasians [7]. Considering the favorable effects of these drugs, DPP-4 inhibitors are frequently added in Japan to treatment regimens that contain basal insulin as the next option for better glycemic control. Furthermore, as vildagliptin strongly inhibits DPP-4 activity by covalently binding to DPP4 [8], this drug might show superior glucose-lowering effects 


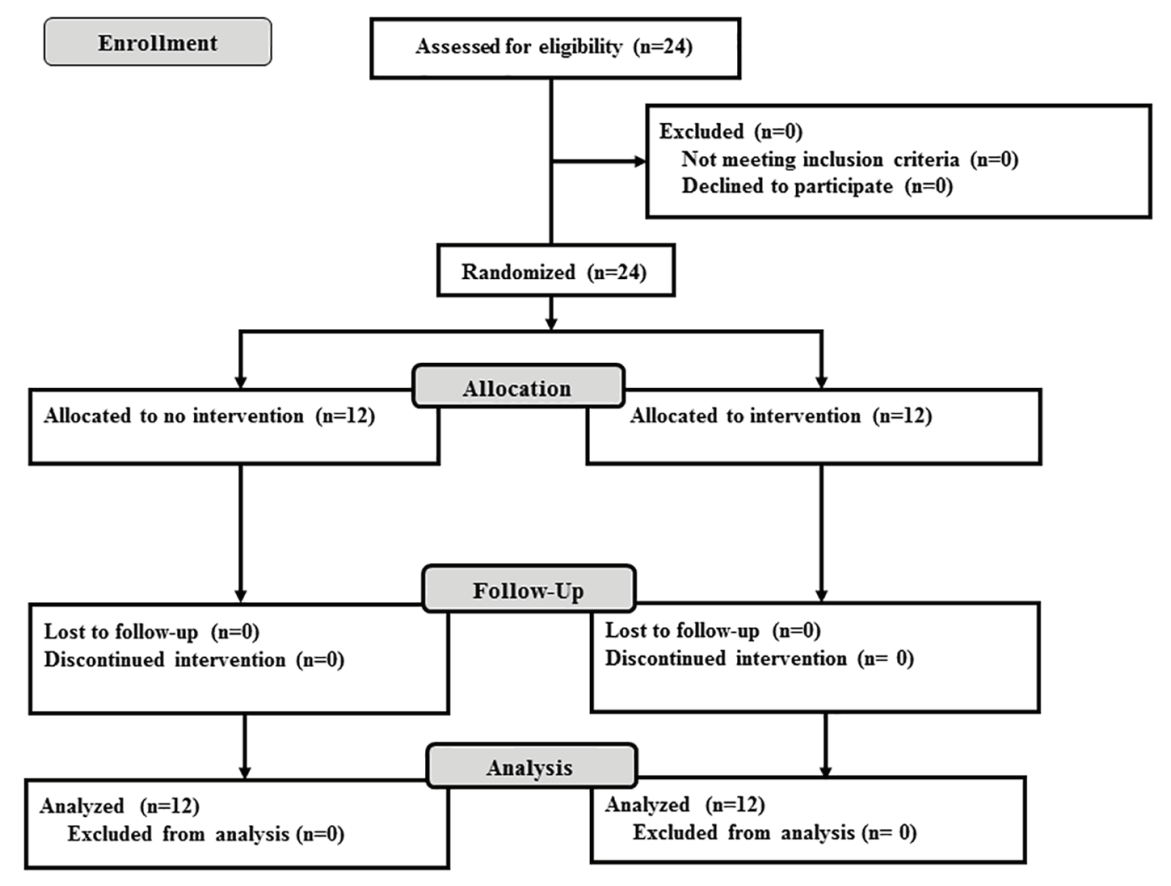

Figure 1. Flow diagram of patient recruitment. Twenty-four patients were randomly allocated to either the control group or the vildagliptin group. All patients were followed up for 6 months.

in uncontrolled patients treated with basal insulin. However, there have been only a few randomized control studies that investigated the efficacy and safety of DPP-4 inhibitors as an add-on drug in patients treated with basal insulin. Therefore, we conducted a 6-month randomized control trial to investigate the efficacy and safety of add-on therapy of vildagliptin. This is the first randomized controlled trial to investigate the effect of vildagliptin, a DPP-4 inhibitor, in Japanese patients with T2DM who were inadequately controlled with basal insulin alone and OADs.

\section{Materials and Methods}

\section{Subjects}

T2DM patients were recruited from the Outpatient Clinic of Juntendo University Hospital, Juntendo Shizuoka Hospital, Tokyo Joto Hospital and International Goodwill Hospital, between May 2013 and April 2015. The following inclusion criteria were applied at study registration: 1) T2DM patients aged $>20$ but $<80$ years; 2) T2DM patients on combination therapy of OADs, excluding DPP-4 inhibitors, and basal insulin alone; and 3) T2DM patients with HbA1c (National Glycohemoglobin Standardization Program: NGSP) $\geq 7.0 \%$ despite treatment of targeting fasting blood glucose (FBG) below 110 $\mathrm{mg} / \mathrm{dL}$ by titration of basal insulin. The selected T2DM patients were excluded from the study if any of the following conditions was diagnosed at registration: 1) proliferative retinopathy, 2) severe neuropathy, 3) serious kidney disease (serum creatinine level $>2.0 \mathrm{mg} / \mathrm{dL}$ ), 4) serious liver disease, 5) acute heart failure, 6) pregnancy, 7) serious infectious disease, 8) trauma injury, 9) pituitary insufficiency, and 10) not suitable for the study. The subjects were screened consecutively, and patients that met the above eligibility criteria were asked to participate in the present study.

The study protocol was approved by the Human Ethics Committee of Juntendo University, and written informed consent was obtained from each patient before enrollment in the study.

\section{Study design}

\section{Determination of sample size}

We performed an open-label, two-arm, randomized controlled study. Vildagliptin is known to reduce HbA1c by $0.6 \%$ with $0.1 \%$ standard deviation (SD) in T2DM patients on insulin treatment [9]. In our study, we adopt $0.5 \% \mathrm{SD}$ considering data variability. With a two-sided $\alpha$ level of $5 \%$ and power $(1-\beta)$ of $90 \%$, at least 24 patients (12 patients in each group) were required to confirm the superiority of vildagliptin in reducing HbA1c. Therefore, we recruited 24 patients who were assigned randomly to either the control group (12 patients) or the vildagliptin group (12 patients) for 6 months. Randomization was achieved by the minimization and biased coin method (Soiken, Inc., Osaka, Japan). The primary endpoint was a change in HbAlc level at the end of the study from the baseline. The secondary endpoint was daily glucose profile by self-monitoring of blood glucose (SMBG). A six-point SMBG (before and 2 $\mathrm{h}$ after the meal) was performed at the baseline and 6 months 
Table 1. Characteristics of Subjects at Baseline

\begin{tabular}{|c|c|c|c|}
\hline & Control & Vildagliptin & P-value \\
\hline $\mathrm{n}$ & 12 & 12 & \\
\hline Gender (males/females) & $8 / 4$ & $11 / 1$ & \\
\hline Age (years) & $61.7 \pm 10.0$ & $60.0 \pm 11.4$ & 0.688 \\
\hline BMI $\left(\mathrm{kg} / \mathrm{m}^{2}\right)$ & $24.8 \pm 5.3$ & $25.6 \pm 5.4$ & 0.719 \\
\hline Systolic blood pressure (mm Hg) & $132.3 \pm 17.7$ & $134.2 \pm 17.4$ & 0.816 \\
\hline Diastolic blood pressure (mm Hg) & $72.3 \pm 13.1$ & $80.1 \pm 11.5$ & 0.183 \\
\hline Diabetes duration (years) & $10.5 \pm 9.1$ & $14.2 \pm 8.8$ & 0.327 \\
\hline HbA1c (\%) & $7.7 \pm 0.6$ & $8.1 \pm 0.7$ & 0.172 \\
\hline $1,5-\mathrm{AG}(\mu \mathrm{g} / \mathrm{mL})$ & $6.4 \pm 3.4$ & $4.4 \pm 3.3$ & 0.183 \\
\hline Fasting blood glucose (mg/dL) & $133.1 \pm 32.3$ & $144.1 \pm 30.1$ & 0.397 \\
\hline C-peptide (ng/mL) & $2.7 \pm 2.5$ & $1.9 \pm 1.1$ & 0.385 \\
\hline C-peptide index & $1.89 \pm 1.46$ & $1.38 \pm 0.76$ & 0.337 \\
\hline Total cholesterol (mg/dL) & $194.3 \pm 35.8$ & $163.9 \pm 26.8$ & 0.056 \\
\hline HDL-C (mg/dL) & $54.9 \pm 21.7$ & $44.0 \pm 12.4$ & 0.158 \\
\hline Triglyceride (mg/dL) & $121.2 \pm 59.9$ & $120.6 \pm 42.2$ & 0.978 \\
\hline UAE (mg/gCre) & $9.8(3.7-78.0)$ & $18.8(12.0-21.8)$ & 0.449 \\
\hline \multicolumn{4}{|l|}{ Medications for diabetes } \\
\hline \multicolumn{4}{|l|}{ Oral anti-diabetes therapy } \\
\hline Sulfonyl urea (n) & 6 & 6 & \\
\hline Metformin (n) & 9 & 9 & \\
\hline$\alpha$-GI inhibitor (n) & 3 & 1 & \\
\hline Thiazolidine (n) & 1 & 2 & \\
\hline Glinide (n) & 0 & 1 & \\
\hline Insulin dose (units) & $9.4 \pm 2.3$ & $10.4 \pm 6.4$ & 0.619 \\
\hline \multicolumn{4}{|l|}{ Insulin type } \\
\hline Detemir & 1 & 2 & \\
\hline Glargine & 8 & 8 & \\
\hline Degludec & 3 & 2 & \\
\hline
\end{tabular}

Data are mean \pm SD or median (interquartile range). BMI: body mass index; HDL-C: high-density lipoprotein cholesterol; UAE: urinary albumin excretion; 1,5-AG: 1,5-anhydroglucitol.

after the beginning of the study using glucometer (Glutest Neo Super $^{\circledR}$, Sanwa Kagaku Kenkyusho, Japan). Otherwise, patients were instructed to measure FBG daily and other blood glucose whenever necessary. The frequency and severity of hypoglycemia, if any, were evaluated during the study period. Severe hypoglycemia was defined as low blood glucose level that required assistance from another person to treat.

\section{Add-on vildagliptin}

In the vildagliptin group, vildagliptin $100 \mathrm{mg} /$ day was added to the current therapy of basal insulin alone. Both groups were observed for 6 months and asked to visit the hospital every month. In both groups, the change in dosage of the current OADs or the addition of new drugs was not allowed during the 6-month study period. However, the dose of basal insulin was adjusted to achieve the target FBG below $110 \mathrm{mg} / \mathrm{dL}$ (110 $\leq \mathrm{FBG}<140 \mathrm{mg} / \mathrm{dL}: 1$ unit increase of basal insulin; FBG $\geq$ $140 \mathrm{mg} / \mathrm{dL}: 2$ units increase of basal insulin), and decrease of basal insulin was judged by each attending physician in order to avoid hypoglycemia.

\section{Biochemical tests}

Blood samples were obtained at overnight fasting. C-peptide index was calculated as follows: serum C-peptide $(\mathrm{ng} / \mathrm{mL}) /$ plasma glucose $(\mathrm{mg} / \mathrm{dL}) \times 100$. Serum total cholesterol $(\mathrm{T}-$ $\mathrm{CHO}$ ), high-density lipoprotein cholesterol (HDL-C), triglyceride (TG), FBG, and HbAlc were measured with standard techniques. 1,5-anhydroglucitol and urinary albumin were 


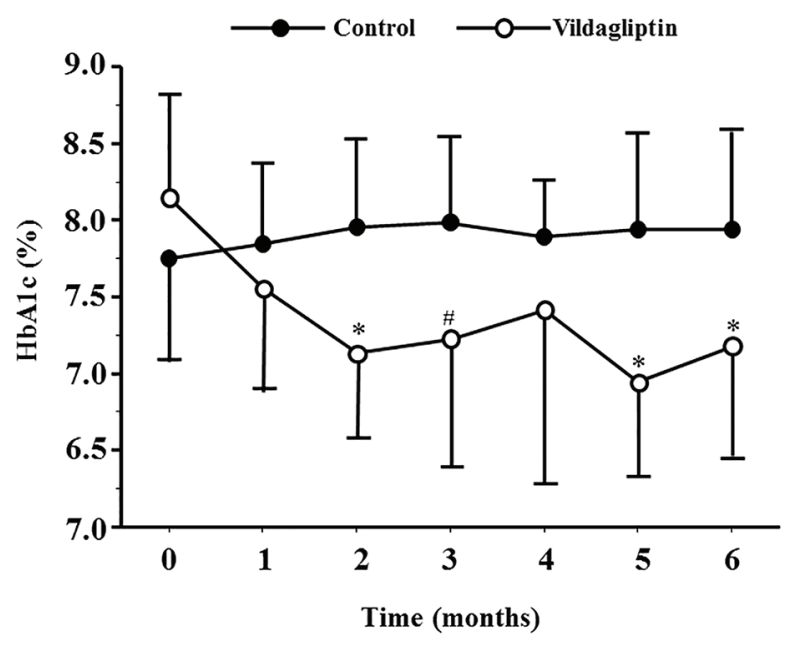

Figure 2. HbA1c levels during the study period in the control and vildagliptin groups. ${ }^{*} \mathrm{P}<0.01$, $\mathrm{P}<0.05$, compared with the control group.

measured by the enzymatic method and immune nephelometric method, respectively. These tests were performed at baseline and after 6-month treatment with vildagliptin.

\section{Statistical analysis}

Data were expressed as mean \pm SD for normally distributed data and median (interquartile range) for data with skewed distribution. The Mann-Whitney $\mathrm{U}$ test and unpaired $t$-test test were used for data analysis. A P value $<0.05$ denoted the presence of a statistically significant difference. All statistical analyses were conducted using StatView statistical software package, version 5.0 (SAS Institute Inc., Cary, NC).

\section{Results}

\section{Baseline characteristics of subjects}

Of the 24 patients recruited in the study, 12 patients were assigned to the control group and 12 to the vildagliptin group. As shown in Figure 1, all patients completed the 6-month study. The baseline characteristics of those patients who completed the study are summarized in Table 1. At baseline, $\mathrm{HbAlc} \mathrm{lev-}$ els in the control and vildagliptin group were $7.7 \pm 0.6 \%$ and $8.1 \pm 0.7 \%$, respectively, and the level was not significantly different between the two groups. The body mass index (BMI) and dosage of basal insulin in the control and vildagliptin groups were $24.8 \pm 5.3$ and $25.6 \pm 5.4 \mathrm{~kg} / \mathrm{m}^{2}$, and $9.4 \pm 2.3$ and $10.4 \pm 6.4$ unit/day, respectively. Again, there were no significant differences in these parameters between the two groups. The data of these baseline characteristics matched those of Japanese patients with T2DM who participated in the ALOHA-2 study [3].

\section{Effects of add-on therapy of vildagliptin on HbA1c and SMBG}

Figure 2 shows the serial changes in HbAlc for both groups. At 2, 3, 5 and 6 months after the start of the study, HbA1c was significantly lower, relative to the baseline, in the vildagliptin group, but not in the control group. The change in $\mathrm{HbA} 1 \mathrm{c}$ from baseline to 6 months after the beginning of the study was significantly higher $(-1.0 \pm 0.3 \%)$ in the vildagliptin group than the control group $(0.21 \pm 0.81 \%, \mathrm{P}<0.01)$ (Fig. 2 and Table 2).

Figure 3 shows the results of SMBG recorded at baseline and study end. In the vildagliptin group, blood glucose levels at $2 \mathrm{~h}$ after breakfast, before dinner and $2 \mathrm{~h}$ after dinner at the end of the 6-month study were significantly lower than those measured at baseline $(215.0 \pm 72.3$ vs. $163.4 \pm 48.3 \mathrm{mg} / \mathrm{dL}$, $\mathrm{P}<0.05,154.6 \pm 56.8$ vs. $102.7 \pm 21.4 \mathrm{mg} / \mathrm{dL}, \mathrm{P}<0.05$ and $207.9 \pm 52.4$ vs. $163.4 \pm 39.9 \mathrm{mg} / \mathrm{dL}, \mathrm{P}<0.05$, respectively). In contrast, blood glucose levels showed no such significant differences in the control group.

\section{Effects of add-on therapy of vildagliptin on 1,5-anhydro- glucitol and other parameters}

In addition to $\mathrm{HbA} 1 \mathrm{c}$, the change in 1,5-anhydroglucitol from baseline to the end of the study was also higher in the vildagliptin group compared to the control group ( $4.5 \pm 3.4$ vs. 0.5

Table 2. Changes in Clinical Parameters Relative to the Baseline

\begin{tabular}{llll}
\hline & Control & Vildagliptin & P-value \\
\hline BMI $\left(\mathrm{kg} / \mathrm{m}^{2}\right)$ & $0.13 \pm 0.44$ & $0.51 \pm 0.81$ & 0.183 \\
Fasting blood glucose $(\mathrm{mg} / \mathrm{dL})$ & $6.5 \pm 24.6$ & $-6.3 \pm 43.6$ & 0.400 \\
C-peptide index $(\mathrm{ng} / \mathrm{mL})$ & $-0.14 \pm 1.09$ & $0.83 \pm 1.54$ & 0.107 \\
UAE $(\mathrm{mg} / \mathrm{gCre})$ & $3.95(-18.5-10.8)$ & $0.4(-6.4-5.1)$ & 0.722 \\
Insulin unit (unit/day) & $1.0 \pm 2.2$ & $2.5 \pm 2.2$ & 0.130 \\
HbA1c $(\%)$ & $-1.0 \pm 0.3$ & $0.2 \pm 0.8$ & $<0.01$ \\
$1,5-\mathrm{AG}(\mu \mathrm{g} / \mathrm{mL})$ & $4.5 \pm 3.4$ & $0.5 \pm 4.1$ & 0.03 \\
\hline
\end{tabular}

Data are mean $\pm S D$ or median (interquartile range). BMI: body mass index; HDL-C: high-density lipoprotein cholesterol; UAE: urinary albumin excretion; 1,5-AG: 1,5-anhydroglucitol. 

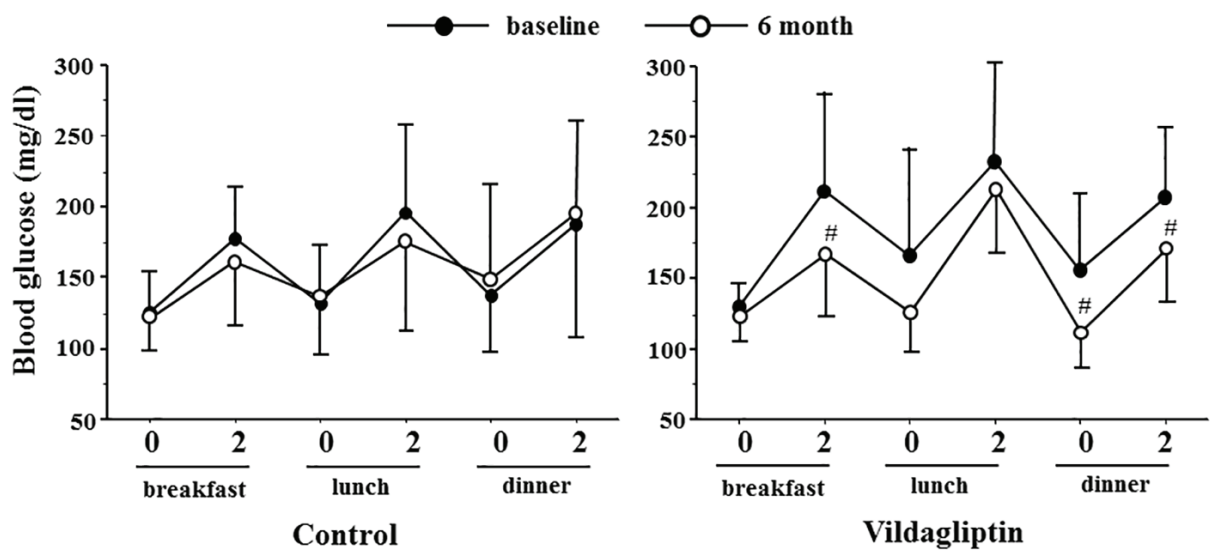

Figure 3. Blood glucose profile in the control and vildagliptin groups measured before $(0 \mathrm{M})$ and after 6 months (6 M). 0 and 2: blood glucose level before and $2 \mathrm{~h}$ after the indicated meal. Vildagliptin significantly improved blood glucose levels $2 \mathrm{~h}$ after breakfast and dinner, and blood glucose levels before dinner compared with the baseline. $\# P<0.05$, compared with the baseline.

$\pm 4.1 \mu \mathrm{g} / \mathrm{mL}, \mathrm{P}=0.03)$. On the other hand, changes in BMI, FBG, C-peptide index, urinary albumin excretion rate and insulin unit were comparable between the two groups (Table $2)$. With regard to the secondary outcome, only one patient of the vildagliptin group and two patients of the control group developed mild hypoglycemia, and this was limited to a single episode in each of these patients. Importantly, no severe hypoglycemia that required assistance by other person and no serious adverse events were reported in both groups during the study period.

\section{Discussion}

The important finding of this study is that add-on $100 \mathrm{mg} /$ day vildagliptin in patients with inadequately controlled T2DM by basal insulin and OADs resulted in significant improvement of glycemic control without increased hypoglycemia. Vildagliptin treatment in our study reduced HbAlc by about $1.0 \%$ from the baseline. This improvement is greater compared to previous studies that investigated the effect of the combination of vildagliptin and insulin $[9,10]$ in Western countries but was similar to that reported in a previous Japanese study [11]. The baseline HbA1c reported in previous studies in Western countries averaged from $8.4 \%$ [9] to $8.8 \%$ [10], which are higher than the baseline $\mathrm{HbA1c}$ levels in our patients. A greater fall in HbA1c could result from treatment of patients with higher baseline HbA1c levels. For this reason, the reduction in $\mathrm{HbA} 1 \mathrm{c}$ recorded in our study was considered high despite the lower baseline HbA1c levels. Considering the greater glucoselowering effect of DPP-4 inhibitors in Asians compared with Caucasians [7], the high efficacy of DPP-4 inhibitors observed in this study is reasonable.

Other reasons for effectiveness of vildagliptin in this study include preserved $\beta$-cell function, as reflected by $\mathrm{C}$-peptide index. Previous studies reported that $\mathrm{C}$-peptide index could predict the importance and need of insulin therapy $[12,13]$. Furthermore, Kozawa et al [14] reported that the mean value of C-peptide index required to maintain good glycemic con- trol after switching from insulin therapy to DPP-4 inhibitors in their in-patient study was $1.5 \pm 0.5 \mathrm{ng} / \mathrm{mL}$. Therefore, $\beta$-cell function of the vildagliptin group (mean C-peptide index: 1.38 $\pm 0.76 \mathrm{ng} / \mathrm{mL}$ ) was considered to be preserved and sensitive to treatment with vildagliptin.

In this study, we investigated the daily glycemic profile by SMBG, and reported that the vildagliptin add-on to basal insulin improved mainly postprandial hyperglycemia, although FBG levels were not different before and after the add-on treatment with vildagliptin. Based on these findings, it can be concluded that the reduction in HbAlc was due to improvement in postprandial hyperglycemia. Indeed, we also observed an increase in 1,5-anhydroglucitol after vildagliptin. Glucose variability is reported to be one of the risk factors for cardiovascular diseases $[15,16]$ and cognitive dysfunction $[17,18]$. Therefore, the combination therapy of vildagliptin and basal insulin is a beneficial option for the treatment of elderly patients with cardiovascular diseases who do not accept intensive insulin therapy and need to avoid hypoglycemia.

\section{Limitations}

Our study has the following limitations; the number of study subjects was too small to confirm the effects of vildagliptin on $\beta$-cell function. Previous report showed that DPP-4 inhibitor increased C-peptide index, and patients with increased Cpeptide index were responders of DPP-4 inhibitor [19]. In our study, the change in C-peptide index after vildagliptin treatment tended to be higher than the control. Therefore, a larger sample size is needed in the future to address this issue.

\section{Conclusions}

Our study showed that $100 \mathrm{mg} /$ day vildagliptin add-on to basal insulin alone improved both glycemic control and glucose fluctuation in Japanese patients with T2DM without increased hypoglycemia. 


\section{Acknowledgments}

We thank all staff of Juntendo University Hospital, Juntendo Shizuoka Hospital, Tokyo Joto Hospital and International Goodwill Hospital for the excellent assistance.

\section{Competing Interests}

AK has received lecture fees from Kissei Pharma, Sanofi and Takeda Pharmaceutical Co. HW has received lecture fees from Asteras, Astrazeneca, Boehringer Ingelheim, Daiichi Sankyo Inc., Eli Lilly and Company, Kissei Pharmaceutical Co., Kowa Pharmaceutical Co., Kyowa Hakko Kirin Co., MSD, Novartis Pharmaceuticals, Novo Nordisk Pharma, Ono Pharmaceutical Co., Mitsubishi Tanabe Pharma, Sanofi-Aventis, Sanwakagaku Kenkyusho, and Takeda Pharmaceutical Co. and research funds from Asteras, Astrazeneca, Bristol-Myers Squibb, Boehringer Ingelheim, Daiichi Sankyo Inc., Dainippon Sumitomo Pharma, Eli Lilly, Johnson and Johnson, Kissei Pharmaceutical Co., Kowa Pharmaceutical Co., Kyowa Hakko Kirin Co., MSD, Mitsubishi Tanabe Pharma, Mochida Pharmaceutical Co., Novartis Pharmaceuticals, Novo Nordisk Pharma, Pfizer, Sanwakagaku Kenkyusho, Sanofi, and Takeda Pharmaceutical Co. All other authors report no conflict of interest.

\section{Grant Support}

None.

\section{References}

1. Home P, Riddle M, Cefalu WT, Bailey CJ, Bretzel RG, Del Prato S, Leroith D, et al. Insulin therapy in people with type 2 diabetes: opportunities and challenges? Diabetes Care. 2014;37(6):1499-1508.

2. Ishii H, Iwamoto Y, Tajima N. An exploration of barriers to insulin initiation for physicians in Japan: findings from the Diabetes Attitudes, Wishes And Needs (DAWN) JAPAN study. PLoS One. 2012;7(6):e36361.

3. Tsukube S, Ikeda Y, Kadowaki T, Odawara M. Improved Treatment Satisfaction and Self-reported Health Status after Introduction of Basal-Supported Oral Therapy Using Insulin Glargine in Patients with Type 2 Diabetes: Sub-Analysis of ALOHA2 Study. Diabetes Ther. 2015;6(2):153-171.

4. Odawara M, Kadowaki T, Naito Y. Effectiveness and safety of basal supported oral therapy with insulin glargine, in Japanese insulin-naive, type 2 diabetes patients, with or without microvascular complications: subanalysis of the observational, non-interventional, 24-week follow-up Add-on Lantus(R) to Oral Hypoglycemic Agents (ALOHA) study. J Diabetes Complications. 2015;29(1):127133.

5. Omar B, Ahren B. Pleiotropic mechanisms for the glu- cose-lowering action of DPP-4 inhibitors. Diabetes. 2014;63(7):2196-2202.

6. Xu L, Man CD, Charbonnel B, Meninger G, Davies MJ, Williams-Herman D, Cobelli C, et al. Effect of sitagliptin, a dipeptidyl peptidase-4 inhibitor, on beta-cell function in patients with type 2 diabetes: a model-based approach. Diabetes Obes Metab. 2008;10(12):1212-1220.

7. Kim YG, Hahn S, Oh TJ, Kwak SH, Park KS, Cho YM. Differences in the glucose-lowering efficacy of dipeptidyl peptidase-4 inhibitors between Asians and non-Asians: a systematic review and meta-analysis. Diabetologia. 2013;56(4):696-708.

8. Ahren B, Schweizer A, Dejager S, Villhauer EB, Dunning BE, Foley JE. Mechanisms of action of the dipeptidyl peptidase-4 inhibitor vildagliptin in humans. Diabetes Obes Metab. 2011;13(9):775-783.

9. Fonseca V, Schweizer A, Albrecht D, Baron MA, Chang I, Dejager S. Addition of vildagliptin to insulin improves glycaemic control in type 2 diabetes. Diabetologia. 2007;50(6):1148-1155.

10. Kothny W, Foley J, Kozlovski P, Shao Q, Gallwitz B, Lukashevich V. Improved glycaemic control with vildagliptin added to insulin, with or without metformin, in patients with type 2 diabetes mellitus. Diabetes Obes Metab. 2013;15(3):252-257.

11. Hirose T, Bailey CP. The "universal" cardiovascular cannula. A tapered corrugated plastic catheter for cannulation in extracorporeal circulation. J Thorac Cardiovasc Surg. 1962;43:559-560.

12. Goto A, Takaichi M, Kishimoto M, Takahashi Y, Kajio H, Shimbo T, Noda M. Body mass index, fasting plasma glucose levels, and C-peptide levels as predictors of the future insulin use in Japanese type 2 diabetic patients. Endocr J. 2010;57(3):237-244.

13. Funakoshi S, Fujimoto S, Hamasaki A, Fujiwara H, Fujita Y, Ikeda K, Takahara S, et al. Utility of indices using Cpeptide levels for indication of insulin therapy to achieve good glycemic control in Japanese patients with type 2 diabetes. J Diabetes Investig. 2011;2(4):297-303.

14. Kozawa J, Kitamura T, Nishizawa H, Yasuda T, Maeda N, Otsuki M, Okita K, et al. Dipeptidyl peptidase-4 inhibitors are effective in Japanese type 2 diabetic patients with sustained endogenous insulin-secreting capacity, a higher body mass index and insulin resistance. J Diabetes Investig. 2013;4(2):190-194.

15. Su G, Mi SH, Tao H, Li Z, Yang HX, Zheng H, Zhou Y, et al. Impact of admission glycemic variability, glucose, and glycosylated hemoglobin on major adverse cardiac events after acute myocardial infarction. Diabetes Care. 2013;36(4):1026-1032.

16. Kataoka S, Gohbara M, Iwahashi N, Sakamaki K, Nakachi T, Akiyama E, Maejima N, et al. Glycemic Variability on Continuous Glucose Monitoring System Predicts Rapid Progression of Non-Culprit Lesions in Patients With Acute Coronary Syndrome. Circ J. 2015;79(10):22462254.

17. Rizzo MR, Marfella R, Barbieri M, Boccardi V, Vestini F, Lettieri B, Canonico S, et al. Relationships between daily acute glucose fluctuations and cognitive performance 
among aged type 2 diabetic patients. Diabetes Care. 2010;33(10):2169-2174.

18. Kim C, Sohn JH, Jang MU, Kim SH, Choi MG, Ryu $\mathrm{OH}$, Lee S, et al. Association between Visit-to-Visit Glucose Variability and Cognitive Function in Aged Type 2 Diabetic Patients: A Cross-Sectional Study. PLoS One. 2015;10(7):e0132118

19. Nishimura T, Meguro S, Sekioka R, Tanaka K, Saisho Y, Irie J, Tanaka M, et al. C-peptide immunoreactivity index is associated with improvement of HbAlc: 2-Year follow-up of sitagliptin use in patients with type 2 diabetes. Diabetes Res Clin Pract. 2015;108(3):441-447. 